\title{
Bodily Feelings and Aesthetic Experience of Art
}

Lauri Nummenmaa ${ }^{1-3}$ and Riitta $\operatorname{Hari}^{4}$

${ }^{1}$ Turku PET Centre, University of Turku, Finland

${ }^{2}$ Department of Psychology, University of Turku, Finland

${ }^{3}$ Turku University Hospital University of Turku, Finland

${ }^{4}$ Department of Art, Aalto University, Finland

\section{Address Correspondence to:}

Lauri Nummenmaa

Turku PET Centre c/o Turku University Hospital

FI-20520 Turku, Finland

Email: latanu@utu.fi

Tel: +35850574 7933

\section{Acknowlegements}

$\mathrm{n} / \mathrm{a}$

\section{Conflicts of interest}

None 


\begin{abstract}
Humans all around the world are drawn to creating and consuming art due to its capability to evoke emotions, but the mechanisms underlying art-evoked emotions remain poorly characterized. Here we show how embodiement contributes to emotions evoked by a large database of visual art pieces. In four experiments, we mapped the subjective feeling space of art-evoked emotions ( $\mathrm{n}=$ 244), quantified "bodily fingerprints" of these emotions ( $n=615)$, and recorded the subjects' interest annotations $(n=306)$ and eye movements $(n=21)$ while viewing the art. We show that art evokes a wide spectrum of emotional feelings, and that the bodily fingerprints triggered by art are central to these feelings, especially in artworks where human figures are the subjectively most salient Altogether these results support the model that bodily sensations are central to the aesthetic emotional experience.
\end{abstract}




\section{Introduction}

Emotion is central to art. Humans all around the world are drawn to creating and consuming art due to its capability to evoke emotions (Chatterjee et al., 2014; Zentner et al., 2008), and human emotions are also a central subject of numerous artworks ranging from The Scream by Edvard Munch to The Kiss by Gustav Klimt. Emotions govern human decision-making across numerous contexts ranging from mate selection (Johnston, 2006) to food (Spence et al., 2016) and environmental preferences (Kaplan, 1987). Emotions act as "survival intelligence" that coordinates physiological and behavioural activation patterns to promote survival. They are often considered as modulatory systems interacting with both physiological control systems, such as allostasis, and higher-order cognitive circuits supporting decision-making (Nummenmaa et al., 2017). Categorical models of emotions propose that evolution has specified a set of basic emotions (usually including anger, fear, disgust, happiness, sadness, and surprise but possibly also others) that support specialized survival functions (Cordaro et al., 2018; Ekman, 1992; Nummenmaa et al., 2017; Panksepp, 1982). These basic emotions are characterised by discrete neural and physiological substrates, distinctive subjective feelings (such as "I feel happy"), bodily and especially facial expressions, and by a specific neural basis (Hudson et al., 2020; Nummenmaa et al., 2017; Wager et al., 2015).

Yet, humans may experience powerful aesthetic emotions in the absence of any survival challenges, most notably when encountering art. Art is an ancient culturally fine-tuned phenomenon. Already homo erectus created primitive ornamentations to their tools (Joordens et al., 2014). The emotions evoked by various forms of art presumably constitute one key factor drawing us to making and viewing visual art and listening to music (see review in Chatterjee et al., 2014; Zentner et al., 2008). However, there is no general consensus whether the emotions associated with art and aesthetic experiences are governed by the same systems that support emotions during survival-salient episodes (Putkinen et al., 2021), and the origins of are-evoked emotions are under intensive debate (Armstrong et al., 2008; Leder et al., 2004; Menninghaus et al., 2019; Skov et al., 2020).

An intriguing yet currently untested hypothesis is that the art-evoked emotions stem from the subjectively felt bodily changes resulting from viewing the art pieces. Human bodies are central to visual art, both as the object of the artwork and as triggers of bodily sensations (such as "touching", "moving"; Kallio-Tavin et al., in press). Previous studies have found that it is human faces and bodies that attract most effectively the viewer's attention in photographs and paintings (Nummenmaa et al., 2012; Pihko et al., 2011). Abundant evidence also suggests that somatosensation and interoception play critical roles in emotion (Craig, 2002; Damasio et al., 2013; 
Nummenmaa et al., 2021). Distinct emotions are associated with discernible self-reported "bodily fingerprints" that are organized in a categorical manner (Nummenmaa et al., 2014; Nummenmaa et al., 2018) and are consistent across a wide range of cultures (Volynets et al., 2019). In line with these findings, the strengths of somatosensory and interoceptive experiences are important determinants of subjective emotional feelings (Nummenmaa et al., 2018) and the aesthetic (versus practical) evaluation of art is associated with activation of the insular cortex (Cupchik et al., 2009), known to be involved in interoceptive processing. It is thus possible that aesthetic experiences evoked by art could be connected to subjectively felt bodily feelings during art encounters. This is particularly likely for artworks containing humans, for which sensorimotor mirroring that supports understanding the socioemotional contents of the depicted scene- However, the role of embodiment in art-evoked emotions remains poorly characterised.

Finally, although low-dimensional ratings of emotions, according to e.g. liking and arousal, can be used for describing emotions emerging while viewing art (Leder et al., 2012), recent work suggests that such conceptualization of aesthetic experiences may be too narrow (Cowen et al., 2020; Cowen et al., 2017; Mohammad et al., 2018). Importantly, the aesthetics-related emotions associated with visual perception and their role in the subjective preferences for specific pieces of art remain poorly characterised. This question is particularly relevant given the mixed and ambiguous emotions evoked by art that people appreciate: Although people generally dislike events and objects that cause negative emotions, various forms of art that evoke negative emotions, such as sadness, may also be strongly liked (Menninghaus et al., 2017; Putkinen et al., 2021).

\section{The Current Study}

Here we investigated embodied emotions evoked by art and their relation to the human figures (faces and bodies) presented in the art pieces. Figure 1 shows the overview of the three experiments, all carried out while the subjects were viewing art. We first mapped the subjective feeling space of art-evoked emotions $(n=244)$ and then quantified the bodily fingerprints of these emotions $(\mathrm{n}=615)$. We then recorded the subjects' interest annotations $(\mathrm{n}=306)$ and eye movements $(n=21)$, trying to find out to which extent the presence of humans in the artworks were associated with the bodily feelings. To ensure generalizability, the stimuli were a large set (n =332) of visual art pieces spanning multiple genres and epochs. 


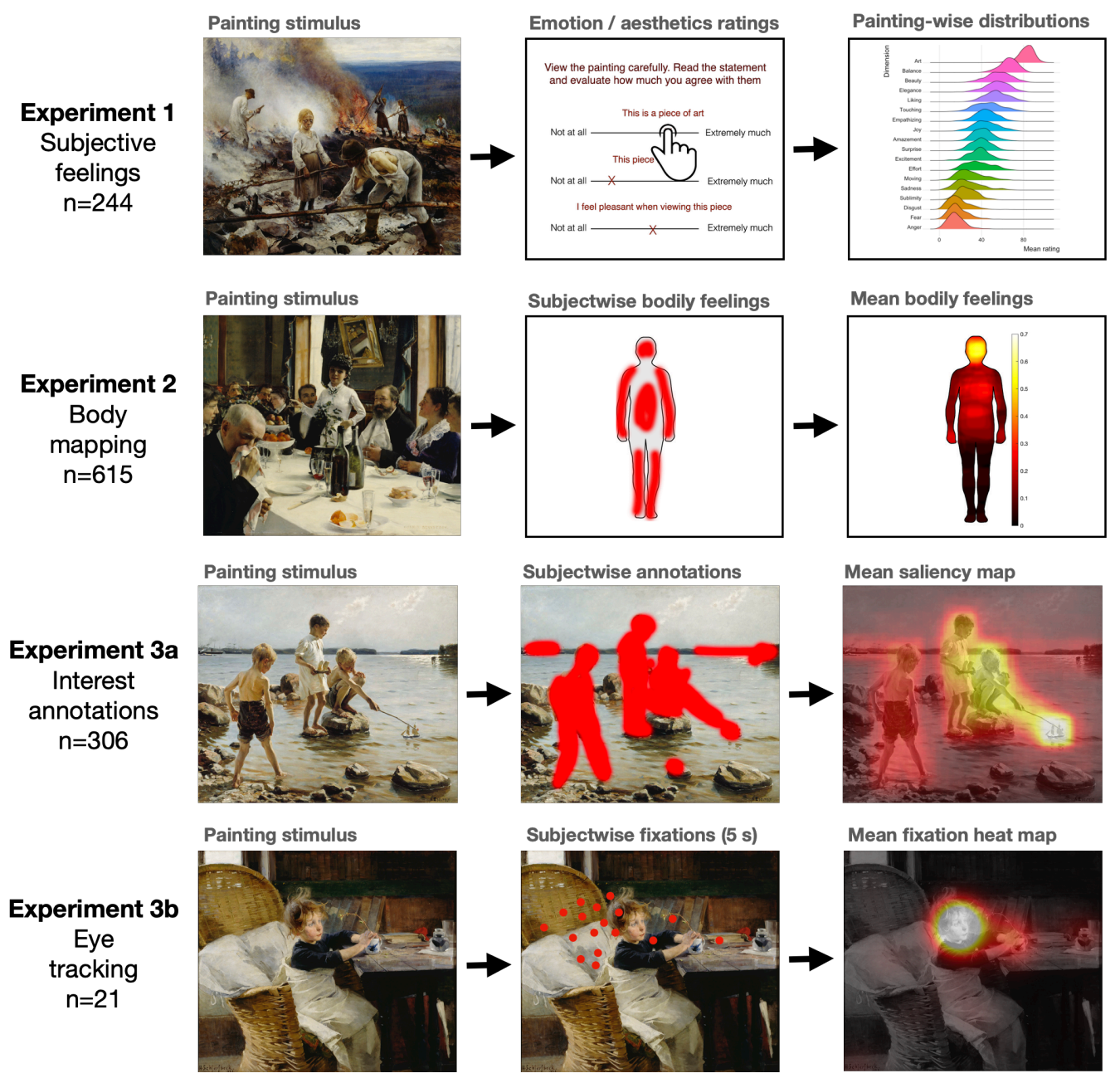

Figure 1. Overview of the study design for Experiments 1-3. Sample paintings from top to bottom: Kaski (Eero Järnfelt), Morsiamen laulu (Gunnar Berndtson), Leikkiviä poikia rannalla (Albert Edelfelt), Toipilas (Helene Schjerfbeck).

\section{Experiment 1: Subjective feelings while viewing visual art}

\section{Materials and Methods}

The stimuli were digital photographs of 336 paintings spanning multiple genres and periods. Most of the stimuli were retrieved from the WikiArt Emotions database and were chosen based on their capacity to evoke emotions in the viewers (Mohammad et al., 2018). These artworks were complemented with 20 internationally famous paintings and 20 famous Finnish paintings (presumably not widely known outside Finland). The stimuli were divided into four broad categories per their content: Portraits $(n=89)$ where single or small groups of individuals portrayed in posed settings, people $(n=145)$ where multiple human figures were represented in various activities, landscapes and objects $(n=33)$ where the nature, environment or objects were the main content and abstract $(n=70)$ works which portrayed no recognizable objects or 
where the level of abstraction in presenting them was high. This categorization should be considered as heuristic content analysis rather than as a fine-grained designation of the art genres; we did not aim at matching the number of paintings in each category.

The experiment was run using Onni online platform developed for measuring bodily sensation maps as well as completing simple questionnaires and preference ratings (Heikkilä et al., 2020). A total of 244 subjects (208 females, 36 males; mean age 40.5 years, SD 18.28 years) volunteered for the study. The experiment contained all the paintings presented in a random order on computer / tablet screen. All images were rescaled to fill approximately $70 \%$ of the screen on their longer axis and presented in a random order. Each painting was evaluated on preference (liking), difficulty (need to struggle to understand the work), art-like qualities (do you consider this art), putative aesthetic dimensions (beauty, balance, touching, capability to evoke empathy) as well as for both basic (happiness, sadness, surprise, fear, anger, disgust) and non-basic (sublimity, amazement, excitement, sublimity) emotions while viewing the work; see Table S-I file for full list of ratings. All ratings were given using visual analogue scale coded from 0 to 100 . The subjects were told to evaluate as many paintings as they wanted, and an average of 22 responses per painting were obtained. We analyzed the data by averaging the ratings for each painting and then computed correlations between the ratings. Hierarchical clustering was used to determine the underlying structure of the rated dimensions, and linear regression model was used for predicting which emotional dimensions determine i) whether a piece was considered as art and ii) how much it was liked.

\section{Results}

Figure 2 shows distributions of painting-wise ratings. Most rated dimensions were relatively normally distributed. Subjects considered the pieces generally as art $(M=82.24, S D=7.61)$ and moderately likable $(M=54.47, S D=11.23)$. They also gave high to moderate ratings on aesthetic dimensions such as balance $(M=65.87, S D=8.14)$, beauty $(M=56.30, S D=11.43)$, elegance $(M$ $=54.91, S D=11.14)$, and touching $(M=46.66, S D=13.23)$. Experiences of surprise $(M=39.69$, $\mathrm{SD}=9.79)$ and amazement $(M=41.40, S D=9.57)$ were moderately common, whereas negative emotions such as sadness $(M=27.87, S D=13.06)$ and anger $(M=15.64, S D=7.91)$ were rare despite many paintings displaying clearly unpleasant scenes (e.g. death, funeral, assault). 


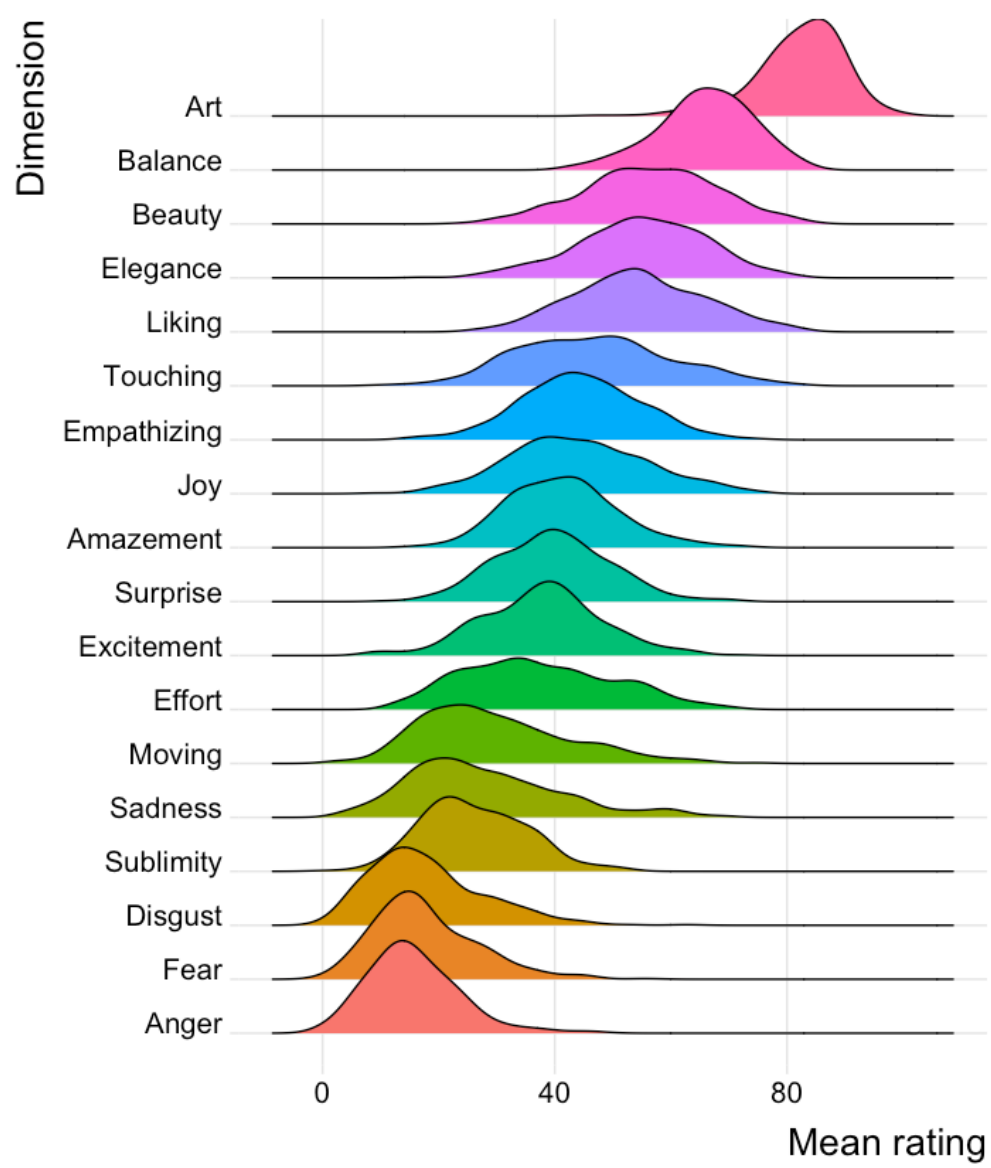

Figure 2. Frequency distributions of the rated dimensions of the art pieces

Correlational and cluster analysis revealed a clear five-cluster structure (Figure 3). Aesthetic dimensions (balance, beauty, and elegance) clustered together and were correlated with the art-like quality of the pictures. Positive emotions (joy, excitement, liking) clustered together with empathizing and sublimity; dimensions in this cluster were also associated with the aesthetic dimensions. Effort needed to comprehend the artwork, amazement, and surprise formed a third cluster. The dimensions in this cluster were also negatively associated with the aesthetic dimensions and positive emotional experiences. The fourth cluster contained negative emotions (fear, anger, disgust), and the final fifth cluster contained sadness and experiences of touching and moving. The negative emotions were negatively linked with the aesthetic and positive emotions, yet the experiences of being touched and moved were positively associated with the aesthetic and positive emotional qualities. 


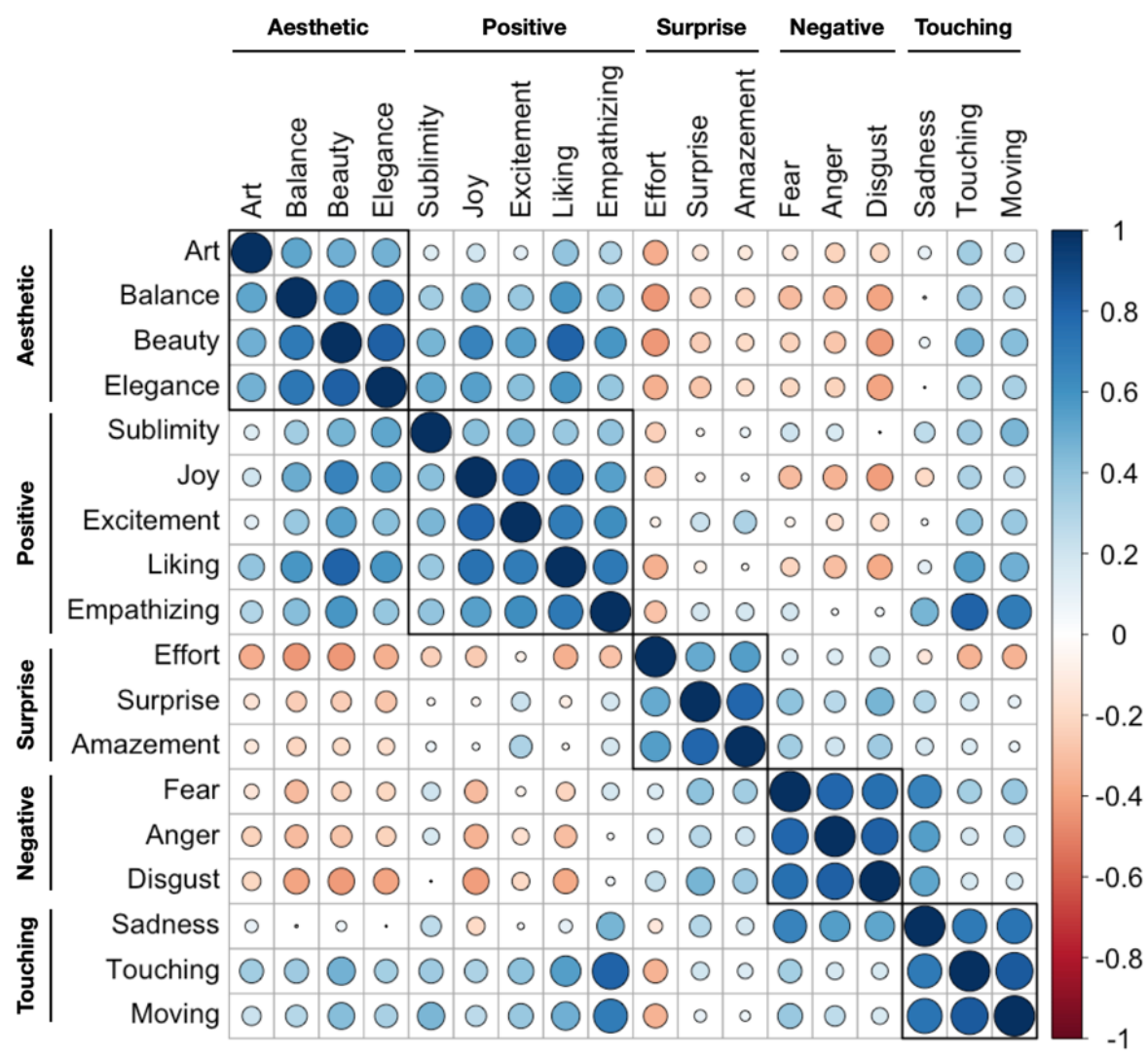

Figure 3. Pearson correlations between the rated dimensions. Black outlines show the clustering of the rated dimensions.

Next, we modelled - using stepwise multiple regression, the dimensions of aesthetic experience that predicted whether a stimulus was i) liked and 2) considered as art. To avoid confounds, liking was excluded from the model where art-like qualities were predicted, and vice versa. Results are summarized in Figure 4. For liking, the model fitted the data well, $F(10,325)=179.20, p<0.001$, and explained a high proportion of the variance, $\mathrm{R}^{2}=0.81$. Of the statistically significant predictors, empathizing, beauty, touching, joy, and excitement had positive regression coefficients (betas), while elegance, fear, and disgust had negative betas. Balance, sublimity, effort, surprise, amazement, anger, sadness and moving were excluded from the model. For art-like qualities, the final model explained the data fitted the data well, $\mathrm{F}(10,325)=23.37, \mathrm{p}<0.001$, and explained almost half of the variance, $\mathrm{R}^{2}=0.42$. Of the statistically significant predictors, beauty, balance, touching, elegance, and amazement had positive betas and effort, joy, anger, excitement and moving negative betas. Empathizing, surprise, sublimity, sadness, and disgust were excluded from the model. 

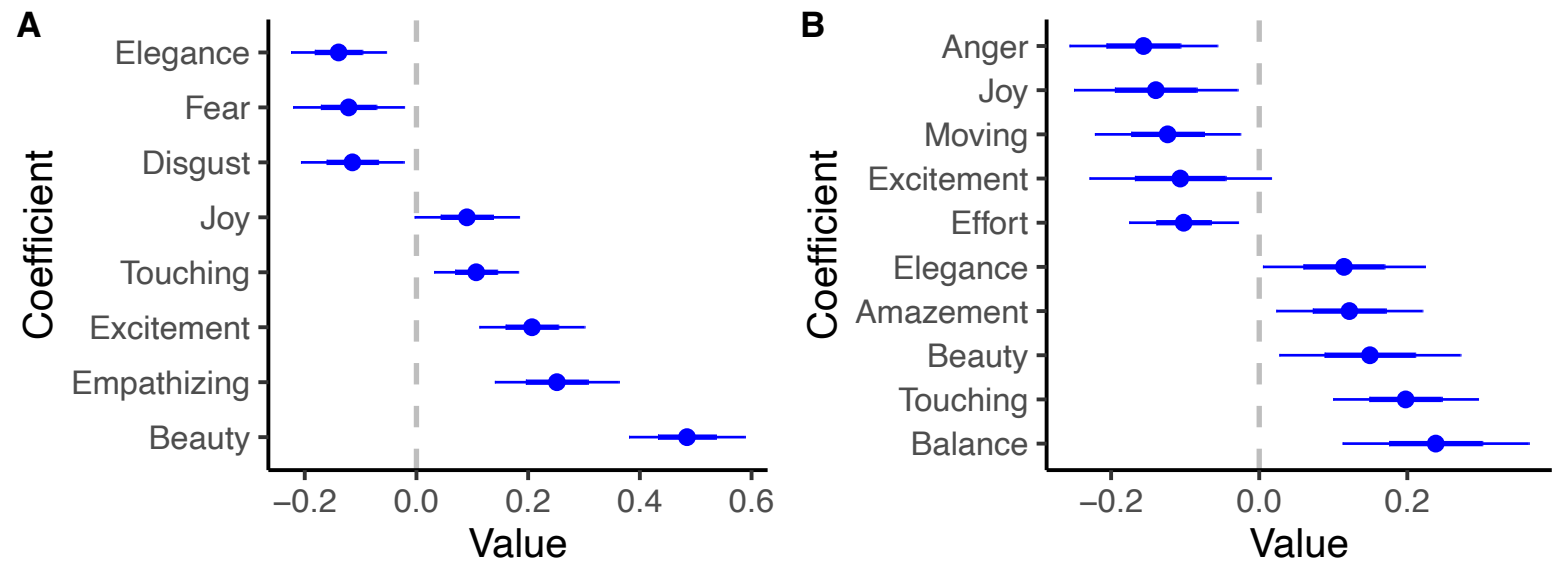

Figure 4. Regression coefficients and their confidence intervals for models predicting art-like-qualities (A) and liking (B) of the artworks in the stepwise regression models. Coefficients are arranged in ascending order separately for each model.

\section{Experiment 2: Bodily feelings while viewing art}

\section{Materials and methods}

We next used the bodily sensation mapping tool developed in our laboratory (Nummenmaa et al., 2014) and tested whether viewing artworks evokes bodily feelings that would be dependent on the emotional and aesthetic dimensions of the art works (as determined in Experiment 1). The stimuli were the same as in Experiment 1. Data were acquired on the Gorilla platform (http://gorilla.sc) and a total of 615 subjects ( 238 females, 377 males; mean age $=26.27$, SD age $=7.62$ ) were recruited from Prolific. Subjects viewed the paintings on a computer / tablet screen one at time. A blank human body was shown alongside each painting. Subjects were asked to view each painting and colour the regions of the body that they felt being activated while viewing the painting. Based on ratings obtained in Experiment 1, the ten paintings with highest score in each dimension (see Figure 2A) were selected for analysis. Individual bodily responses were averaged across subjects for the ten paintings for each dimension, and pixelwise mass univariate t-tests were conducted to reveal statistically significant activations for each painting. Finally, false discovery rate correction (Benjamini et al., 1995) was applied to control for false positives.

To assess the link between bodily feelings and emotional and aesthetic evaluations of art, we computed the average number of pixels coloured in the human figures for each painting. These indices, calculated separately for the head and body regions, range from 1 (every subject coloured every pixel) to 0 (no subject coloured any pixel). On the basis of our previous work (Nummenmaa et al., 2018), we considered colouring of the body region to imply embodiment or bodily component of the sensations, whereas colouring of the head region was considered to imply 
mentation, here called mental component. The indices were subsequently correlated with the painting-wise ratings from Experiment 1 to test how the bodily and mental components of feelings link with the consciously accessible emotions while viewing the paintings. Because embodiment might be affected, via mirroring mechanisms, on seeing other humans in the paintings, we also tested whether embodied and mental components would vary across painting types (portraits, people landscapes, objects, and abstract paintings).

\section{Results}

Figure 5 shows the bodily feeling maps elicited by viewing art. On average, the strongest bodily feelings were associated with empathizing, fear, anger, elegance, and joy. Most of the evaluated dimensions were associated with bodily sensations in the chest area, while feelings in the upper limbs were most salient for anger, empathizing, and fear. Experiences of amazement and effort were markedly lacking in the embodied sensations.

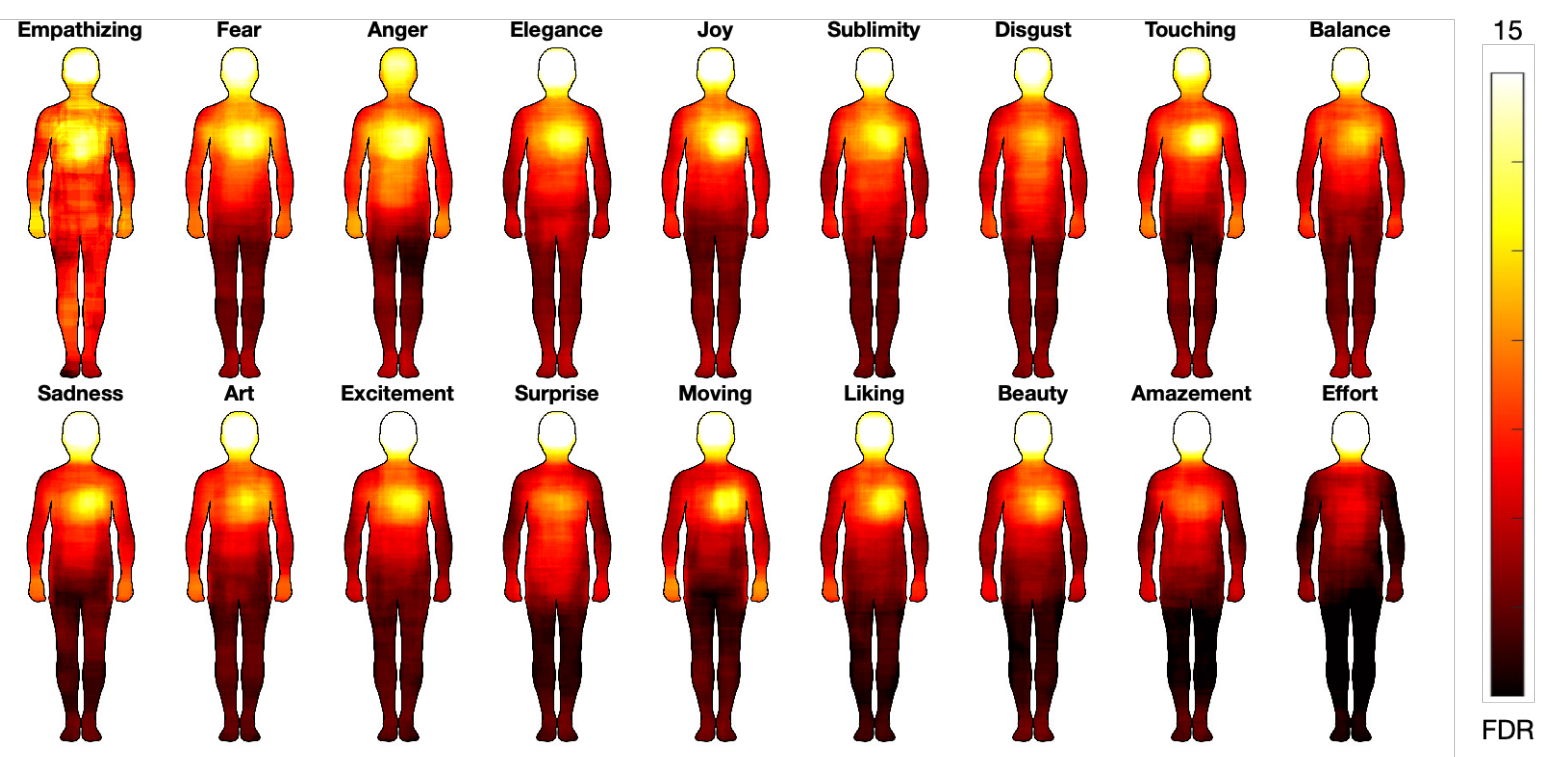

Figure 5. Bodily maps of aesthetic and emotional experiences while viewing art. The maps show the statistically significantly activated regions across subjects for each feeling. The maps are arranged as a function of the mean proportion of significant pixels, and thresholded at $p<0.005$, FDR corrected.

Bodily and mental components were negatively correlated $(\mathrm{r}=-0.28, \mathrm{p}<0.001)$. Strength of the bodily component (Figure 6 and Table S-2) was positively correlated with experiences of touching, empathizing, moving, sadness, fear, beauty, art, anger, elegance and liking (r:s $>0.11$, ps $<0.05$ ) and negatively correlated with experiences of amazement and effort ( $\mathrm{rs}<0.12$, ps $<0.05)$. 
Mental components showed significantly sparser pattern of associations, with positive correlations for excitement, surprise, and amazement (r:s $>0.16$, ps $<0.05)$. Finally, we observed that bodily feelings varied across painting types, $F(3,332)=30.06, p<0.001$. Bodily sensations were strongest for pictures with people, and the associated bodily sensations differed statistically significantly from those for portraits or those with abstract content ( $\mathrm{p}: \mathrm{s}<0.001)$. The sensations were second strongest for landscapes, which differed from abstract paintings $(\mathrm{p}<0.001)$, but not from paintings with people or portraits (Tukey's HSD test). Mental component also varied as a function of painting type $\mathrm{F}(3,332)=4.76, \mathrm{p}=0.002$, although less profoundly than for bodily sensations. Mental sensations were stronger for paintings with abstract content versus people, $p=0.005$, and for portraits versus people, $\mathrm{p}=0.026$ (Tukey's HSD test); no other statistically significant differences were found.

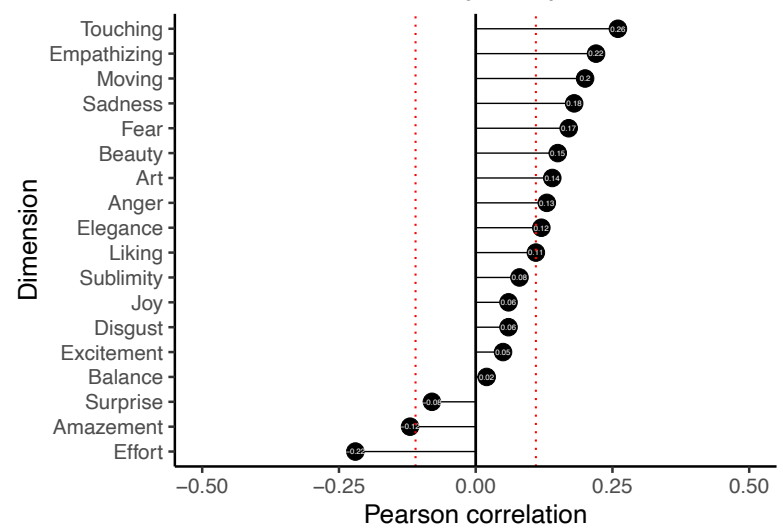

B Correlation with mental component

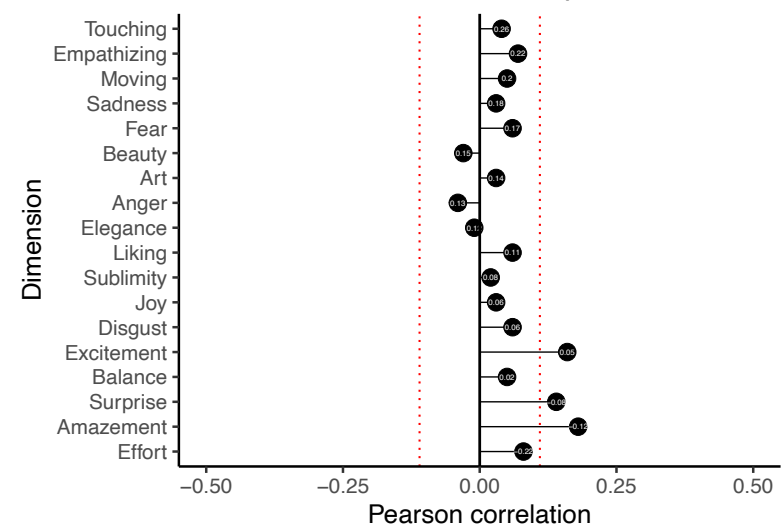

Figure 6. Bodily and mental components and aesthetic and emotional evaluations of art. Mean Pearson correlation between bodily (A) and mental (B) sensations and the aesthetic and emotional qualities of the paintings. Red lines show the area of nonsignificant correlations.

\section{Experiment 3: What interests us in paintings?}

Experiment 2 revealed that bodily sensations are key determinants of emotional responses to art. It further indicated that art pieces with human figures evoked the strongest bodily sensations. Thus, the presence and saliency of humans in the artworks might enhance attention and interest towards the paintings, thus being a potential mediator for art-mediated emotions. To measure the spatial distribution of such top-down saliency or interest in the art pieces and link them with the affective evaluations, we next used human "interest annotations" in a subset of the paintings ( $\mathrm{n}=$ 60) used in Experiment 1.

\section{Materials and methods}


Data were acquired on the Gorilla platform (http://gorilla.sc) and a total of 306 subjects (134 females, 172 males; mean age $=26.35$, SD age $=7.04)$ were recruited from Prolific. Subjects viewed the paintings on a computer / tablet screen one at time and were asked to colour the most interesting areas from the painting. No specific instructions for what consist of "interesting" were given, the subjects were simply asked to act based on their intuition and gut feelings. The image batch was divided into half, and each subject annotated 30 paintings. Painting data were stored as binary (paint vs. no paint) matrices and mean proportion of subjects colouring each pixel in each artwork was computed for generating 2D subjective saliency maps were then analysed. Mean, SD, skewness, kurtosis, RMS contrast as well as the number of peaks in each map were also computed to characterise the spatial distribution of the saliency annotations. Additionally, we computed mean intrasubject similarity (based on Spearman correlation) for the painting-specific interest annotation maps to index consistency of the saliency annotations across subjects. To quantify the content of the interest annotation maps, we thresholded the maps at $>0.33$, corresponding to one third of the subjects annotating a pixel as interesting. We then counted the number of interest clusters in each image and counted the number of the resulting clusters. Finally, we categorized the broad content of each cluster (faces, whole human bodies, human body parts (e.g. hands), objects, animals, and landscapes). Each cluster could receive up to three content labels, for example one cluster could contain both human face and human body parts)

To validate the interest annotation approach and to benchmark visual attentional engagement while the subjects were viewing art, we also conducted an eye movement study. Gaze position reliably indexes direction of attention and subsequently provides a net index of both task-relevant and visually salient locations in a scene (Henderson, 2003). We presented the same stimuli as used in the interest-annotation experiment to 21 observers (13 females, 8 males; mean age 33.6 years, $\mathrm{SD}=8.5)$ while their eye movements were recorded using Eyelink 1000 tracker (SR Research) at $1 \mathrm{kHz}$ sampling rate. Spatial accuracy was better than 0.5 degrees of visual angle. Stimuli were rescaled to 1200 pixels in height and were shown on a 27" screen for 5 seconds each. Subjects were instructed to view the paintings as they would view images on a computer or tablet screen. We first extracted mean number of fixations and blinks and, average saccade amplitude and pupil size for each trial, and compared these parameters across the a priori painting categories. Subjectwise fixation heatmaps were generated and averaged across subjects for each painting and compared with the interest annotation maps using Spearman correlations. To address the latency at which the manual annotations and eye-movement-based saliency maps coincide, we 
reconstructed the fixation heatmaps with 1-20 first fixations on the image and computed the similarity index between each reconstruction and the manual annotations.

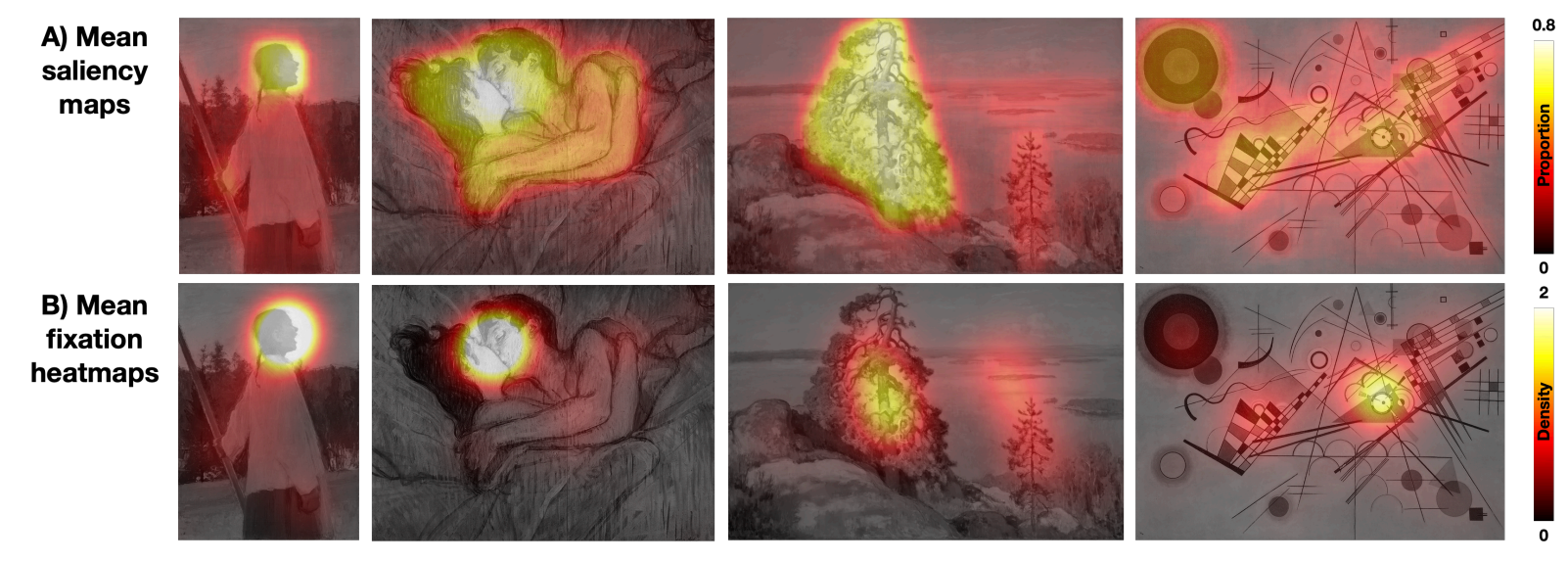

Figure 7. Representative manually annotated mean saliency maps (A) and fixation beatmaps (B) for 4 representative paintings. Colourbars show the proportion of subjects colouring each area (top) and the density estimate (B). Sample paintings from left to right: Kaiku (Ellen Thesleff), Dans le Lit, le Baiser (Henri de ToulouseLautrec), Maisema Kolilta (Eero Järnfelt), Composition 8 (V assily Kadinsky).

\section{Results - Interest Annotations}

Figure 7 shows representative manually annotated interest maps. Visual inspection of the saliency maps revealed that the overall consistency in the saliency annotations across observers was high, that there were one or two clear "hotspots" of annotations for each painting with relatively low saliency ratings in the background, and that the annotations tended to focus on humans whenever those were present in the paintings. These features were confirmed by the formal analysis. On average, the subjects annotated 2.35 regions per image (Figure 8A). Faces were the most commonly interesting target, followed by objects, bodies, and body parts, whereas landscapes and animals were annotated relatively rarely. Mean consistency of the annotations (as indexed by intersubject correlation, ISC) was $r=0.42$, confirming that the subjects agreed well with each other regarding the most interesting regions in the paintings (Figure 8B). 

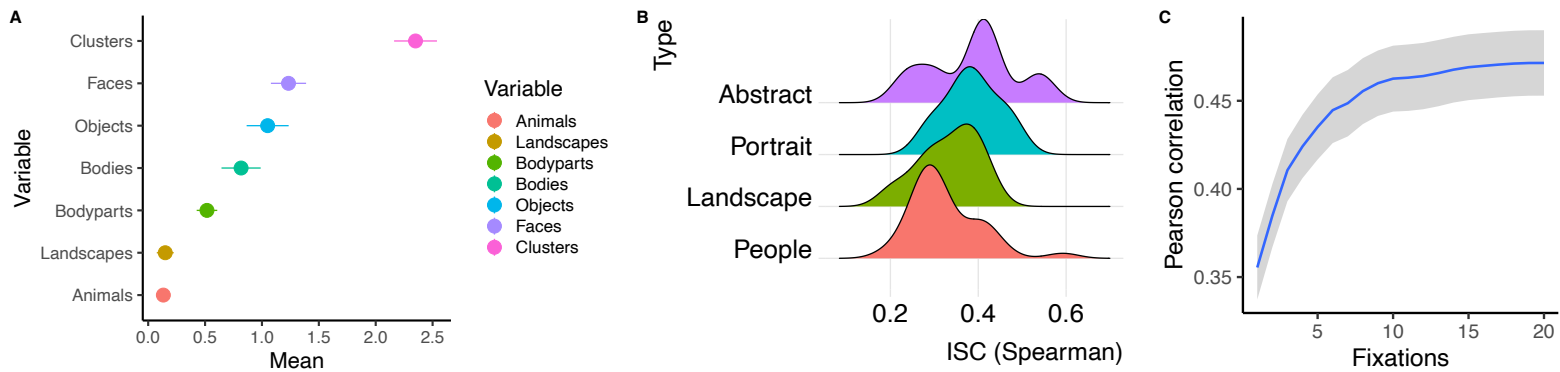

Figure 8. Summary of the annotation results. Means \pm standard errors of mean for the number of clusters and the frequency of the contents for the clusters (A). Distribution of intersubject similarity of the painting annotations (n=336) for different painting types (B). Mean \pm standard error of mean intsersubject correlation (Spearman) between the interest annotations and gaze-based saliency maps from 1-20 first fixations (C).

\section{Results - Eye Tracking}

Figure 7B shows mean fixation heatmaps for 4 representative paintings, and video S-1 shows mean eye movement patters while our 21 subjects were viewing a subset of the artworks. On visual inspection, the interest-annotation maps (Figure 7A) matched well with the fixation heatmaps. This consistency was confirmed with formal statistical analysis, showing that the mean Spearman correlation between the fixation heatmaps and interest annotations was $0.49(S D=0.13)$ when computed over the whole 5-s trial period. The correspondence between interest annotations and fixation heatmaps increased as a function of time (Figure 8c). Table 1 shows correlations between eye movement parameters and ratings of the aesthetic / emotional dimensions of the paintings. This metric plateaued around 10 fixations (corresponding to $\sim 4$-s viewing time as mean fixation duration was $402 \mathrm{~ms}$ ). Fixation count and duration were most consistently associated with the aesthetic and affective dimensions so that fixation count decreased as a function of art-like quality, beauty, balance, sadness, and moving $(r s>036, p s<0.05)$. Fixation duration increased as a function of the same variables, except that additional correlation was observed with touching ( $r$ s $>036, p s<0.05)$. Blink rate was positively associated with joy and negatively with disgust ( $r s>$ 036, $p$ s < 0.05). Pupil size was positively associated with negative emotions of sadness, and fear, anger, and disgust, and negatively with joy $(r \mathrm{~s}>036$, $p \mathrm{~s}<0.05)$.

Table 1 Correlations (Pearson) between eye movement parameters and aesthetic and affective dimensions of art. Statistically significant correlations $(p<0.05)$ are shown in boldface.

\begin{tabular}{lccccc}
\hline \multirow{2}{*}{ Dimension } & Fixation & Fixation & Saccade & Blink & \\
& count & duration & amplitude & count & Pupil size \\
\hline
\end{tabular}




\begin{tabular}{lccccc}
\hline Liking & -0.20 & 0.21 & 0.01 & 0.21 & -0.14 \\
Art & -0.31 & $\mathbf{0 . 3 1}$ & -0.14 & 0.09 & 0.19 \\
Effort & 0.19 & -0.16 & -0.03 & -0.19 & 0.00 \\
Empathizing & -0.15 & 0.21 & -0.08 & 0.08 & 0.06 \\
Beauty & -0.32 & $\mathbf{0 . 3 1}$ & -0.09 & $\mathbf{0 . 2 4}$ & -0.06 \\
Balance & -0.30 & $\mathbf{0 . 3 3}$ & -0.17 & 0.17 & 0.04 \\
Touching & -0.23 & $\mathbf{0 . 2 8}$ & -0.10 & 0.07 & 0.15 \\
Elegance & -0.25 & $\mathbf{0 . 2 5}$ & -0.09 & 0.14 & -0.09 \\
Joy & -0.09 & 0.07 & 0.12 & $\mathbf{0 . 2 7}$ & -0.34 \\
Sadness & -0.28 & $\mathbf{0 . 3 4}$ & -0.06 & 0.03 & $\mathbf{0 . 3 3}$ \\
Surprise & 0.08 & -0.03 & -0.02 & $\mathbf{- 0 . 2 5}$ & 0.12 \\
Fear & -0.20 & 0.21 & -0.17 & 0.02 & $\mathbf{0 . 3 5}$ \\
Anger & 0.05 & -0.02 & -0.04 & -0.10 & 0.28 \\
Disgust & 0.17 & -0.11 & -0.15 & $\mathbf{- 0 . 3 0}$ & 0.27 \\
Sublimity & -0.20 & $\mathbf{0 . 2 3}$ & 0.07 & 0.11 & -0.05 \\
Amazement & -0.08 & 0.10 & 0.10 & -0.02 & 0.02 \\
Excitement & -0.07 & 0.05 & 0.15 & $\mathbf{0 . 2 5}$ & $\mathbf{- 0 . 2 1}$ \\
Moving & -0.29 & 0.35 & -0.07 & $\mathbf{0 . 2 3}$ & 0.14 \\
\hline
\end{tabular}

Figure S-1 shows mean trial-wise eye movement parameters arranged by painting type. Number of fixations varied as a function of painting type, $F(3,56)=5.34, p=0.003$. Subjects made more fixations on landscape paintings and paintings with people than on portraits $(p s<0.01)$. Saccade amplitudes also varied as a function of painting type, $F(3,56)=4.58, \mathrm{p}=0.003$. Subjects made longer saccades on landscape versus portrait paintings $(p=0.004)$. Blink counts varied as a function of painting type, $\mathrm{F}(3,56)=3.93, \mathrm{p}=0.01$. Subjects blinked more when viewing portrait paintings than paintings with people $(p=0.05)$. Pupil size varied as a function of painting type, $\mathrm{F}(3,56)=7.53$, $\mathrm{p}<0.001$, being largest for portraits and second-largest for paintings with people, which differed from landscape paintings $(\mathrm{p}=0.004)$. Pupil size was larger for portraits than that for landscape or abstract paintings $(p s<0.01)$.

\section{Discussion}

We found that viewing visual art pieces evokes a wide range of emotional experiences that formed five broad clusters: (1) aesthetic experiences, (2) positive emotions, (3) negative emotions, (4) touching feelings, and (5) feelings of surprise and effort. These dimensions could be reliably used 
for predicting how much individual artworks were liked and whether the works were considered as art in the first place. These emotion dimensions were strongly embodied, meaning that the subjects reported clear bodily feelings associated with them. Empathy, anger, fear and elegance evoked the strongest bodily feelings in mean maps across subjects, whereas liking, beauty, amazement and effort elicited the weakest. The strengths of the bodily feelings were consistently associated with emotions experienced while viewing art. Finally, both manual interest annotation and eye-gaze maps confirmed that human faces and bodies were consistently the most interesting features in the paintings, and that the presence of humans in the paintings increased pupil size, indicative of affective arousal. Altogether these studies reveal that visual art evokes strong embodied experiences, and that the bodily feelings are an important determinant of the aesthetic emotional experience.

\section{Emotional encounters with visual art}

Viewing art was associated with strong emotional experiences that were consistent in our large sample of observers. Aesthetic emotions (art, balance, beauty, and elegance) were most prominent, followed by positive emotions (liking, empathizing and joy) and empathy. Feelings linked with surprise and effort were moderately common. Finally, any sort of negative emotions was rare although numerous paintings contained negative themes, such as sad, scary, and disgusting events. Some negative emotions were commonly experienced with the aesthetic, non-basic emotions. In particular, sadness was consistently associated with the experience of being touched and moved by the artworks, although these emotions were also consistently associated with pleasant emotions such as joy. This brevity of self-reported feelings go significantly beyond simple liking and arousal (Leder et al., 2012) as well as the putative "basic" emotions (Cordaro et al., 2018; Ekman, 1992; Nummenmaa et al., 2017; Panksepp, 1982). It rather accords with the view that mixed positive and negative emotions are a common feature of the aesthetic emotional experiences during art encounters (Menninghaus et al., 2019).

The consistent cluster structure of the art-evoked emotions in our data distinguished between the positive and negative emotions, but also the aesthetic and surprising dimensions and the touching qualities of the feelings. The aesthetic dimensions (art, balance, beauty, and elegance) were consistently associated with the positive emotions (sublimity, joy, excitement, liking and empathizing) and negatively associated with the experience of surprise, effort, and negative emotions (fear, anger, disgust). However, considering the art pieces touching and moving were positively associated with aesthetic experiences, as was also to some extent sadness. All in all, these data suggest that constellation of aesthetic experience is generally positive in nature. 
The aesthetic feelings also contributed significantly to liking of the art pieces: the more empathy, beauty, touching, joy, and excitement a painting elicited, the more it was liked. Conversely, particularly the negative emotions (fear and disgust) were negatively linked with liking of the art. Importantly, beauty and touching also predicted whether or not a piece was considered as art, suggesting that these feelings have a key role in determining the ultimate aesthetic response to visual art. In line with these subjective reports, functional imaging experiments suggest that empathetic responses could be important mediators of aesthetic experiences, in addition to those linked with evaluation of beauty (Ardizzi et al., 2021).

Although negative emotions are often argued to increase the enjoyment or likability of artworks (Menninghaus et al., 2017), the present data suggest the opposite. Negative emotions may play an important role in the aesthetic experience, for example via the embodied feelings they evoke, and in our study, particularly anger and fear were consistently associated with strong bodily feelings (see Figure 5-6). These emotions do not however increase the preference for the art piece or their subjective art-like qualities. It is thus possible that negative-emotions-eliciting events commonly depicted in visual art elicit strong "thrill-seeking" -related visceral states, which however do not manifested in the subjective likability of the works.

Finally, likability of art was negatively associated with the effort required to comprehend the work. Although humans associate effort with reward, they also consider reward as costly and avoid it on many occasions (Inzlicht et al., 2018). The present data suggest that in the context of art, effort is on average considered as an extra cost associated with the aesthetic experience, thus decreasing the likability of the works. However, appreciating the effort in comprehending an artwork may be strongly dependent on the qualities of the individual viewers, especially their history of art encounters and expertise and experiences of the specific art style, and this issue needs to be addressed in future studies.

\section{Embodied emotions and art}

Art-evoked emotions were accompanied with strong bodily feelings, confirming that art perception is not only an exteroceptive but also a strongly interoceptive process. Unlike bodily feelings evoked by survival-salient episodes depicted in movies (Nummenmaa et al., 2014), the bodily signatures of aesthetic emotions revealed a continuum from the whole-body experiences of empathy, fear, and anger towards the experiences of beauty, amazement and effort that were mostly reported in the head area, possibly reflecting cognitive processing. Although the self-report technique cannot discern the underlying brain activation patterns, it is noteworthy that the relative unspecificity of the bodily signatures accords with brain activation patterns recorded during 
aesthetic emotions. While pattern recognition studies have established that a wide range of emotions elicited by biologically salient events are associated with discernible cortical and subcortical activation patterns (Kragel et al., 2016; Saarimäki et al., 2018; Saarimäki et al., 2016), a similar "decoding" of music-evoked aesthetic emotions did not work outside the sensory (and motor) cortices (Putkinen et al., 2021). It is thus possible that the emotions evoked by art and other abstract events and objects only engage the low-level pleasure/ displeasure and arousal dimension of emotion circuits, even though the resultant subjective experience might be affectively vivid and complex.

The stronger the bodily responses for a given art piece, the stronger were the subjectively felt emotional feelings. This effect was strongest for feelings of touching, empathy and moving, and also statistically significant for sadness, fear, beauty, art, anger, elegance and liking,. Instead, experiences of amazement and effort correlated negatively with the bodily sensations. This finding accords with the models of emotional experiences that posit an important role of interoception and somatosensation in the emotional experience (Craig, 2002; Damasio et al., 2013), and also with work showing a tight linkage between bodily feelings and emotional experience (Nummenmaa et al., 2018). Because experiences of touching and empathizing were significant predictors of whether a piece was considered as art, it is possible that this kind of bodily sensations are an integral part of the subjective evaluative processes when viewing art.

\section{What interests the viewers in art?}

Other people are our most important "environment", and the human brain has been finely tuned for processing of social information (Hari et al., 2015; Hari et al., 2009). This profound interest in conspecifics is also reflected in the common themes in visual arts, and a large bulk of the art pieces studied here represented humans in somewhat realistic or at least recognizable form. The interest annotation (Experiment 3a) revealed that human faces were most annotated as interesting, followed by objects and human bodies. Backgrounds received practically no annotations. This strong preference for faces accords with prior eye tracking work on photographs and art pieces depicting humans (Nummenmaa et al., 2012; Pihko et al., 2011). Subjects were consistent in their annotations, with mean intrasubject correlation of $r=0.42$. Thus, despite the potentially complex visual structure of the presented art work, most pieces conveyed their interesting features similarly to all viewers. The manual interest-annotation maps correlated significantly with eye-movementbased heatmaps, according with the view that eye movements during natural vision reflect both low-level bottom-up visual features as well goal-relevant information (Henderson, 2003), such as the features that subjects consider interesting. Prior studies have shown that pupil dilation reflects 
not only background luminosity, but also emotional arousal and autonomic activation (Bradley et al., 2008; Nummenmaa et al., 2012). Accordingly, we found that pupil size was positively correlated with the negative emotions (sadness, anger, fear, and disgust) evoked by the paintings. The aesthetic experiences evoked by the art pieces were, in turn, associated with significantly longer fixations, indicating stronger attentional engagement (Nummenmaa et al., 2006). Finally, saccade durations were different particularly for portrait and landscape paintings, indicative of differneces in focal versus ambient processing of paintings with different contents (Pannasch et al., 2009).

\section{Limitations}

Although our stimulus set was sizable $(\mathrm{n}=334)$ and variable with respect to art style and historical period (ranging from years 1400 to 1900), it obviously does not cover the whole spectrum of what could be considered as visual art across human cultures. Also, we did not explicitly control for the subjects' familiarity with the presented artworks. However, we deliberately chose only a limited number of famous / well-known paintings in the dataset. We also focused on self-reported emotional experiences and bodily feelings, rather than direct psychophysiological recordings. Although interoception allows tracking of internal bodily states (Critchley et al., 2004), self-reports such the bodily sensation maps employed here cannot provide one-to-one mapping with specific physiological activation patterns. Consequently, our data cannot reveal whether the presently measured aesthetic emotions distinct from physiological or neural affective states during other type of sensory perception (see e.g. discussion in Menninghaus et al., 2019; Skov et al., 2020)

Nevertheless, our data reveal that encountering visual art is associated with a wide variety of "basic" and non-basic aesthetic subjective experiences. Our bodily mapping technique further provides a net index of the current, consciously accessible states of various physiological systems in the body, thus constituting an important part of the emotional experience (Nummenmaa et al., 2018).

\section{Conclusions}

We conclude that aesthetic emotional experience associated with encountering visual arts are strongly embodied and that visual arts can elicit a broad range of emotional feelings that go significantly beyond the canonical "basic" emotions. The strength of these emotions is, in general, linked with the strength of bodily sensations the art pieces evoke. Accordingly, it is possible that the appeal of the visual arts stems partially from art's capability to engage the viewer's body in a manner resembling the bodily signatures of survival-salient emotions. 



\section{References}

Ardizzi, M., Ferroni, F., Umiltà, M. A., Pinardi, C., Errante, A., Ferri, F., . . Gallese, V. (2021). Visceromotor roots of aesthetic evaluation of pain in art: an fMRI study. Social Cognitive and Affective Neuroscience. doi:10.1093/scan/nsab066

Armstrong, T., \& Detweiler-Bedell, B. (2008). Beauty as an Emotion: The Exhilarating Prospect of Mastering a Challenging World. Review of General Psychology, 12(4), 305-329. doi: $10.1037 / \mathrm{a} 0012558$

Benjamini, Y., \& Hochberg, Y. (1995). Controlling the false discovery rate - A practical and powerful approach to multiple testing. Journal of the Royal Statistical Society Series BMethodological, 57(1), 289-300. Retrieved from <Go to ISI>://WOS:A1995QE45300017

Bradley, M. M., Miccoli, L., Escrig, M. A., \& Lang, P. J. (2008). The pupil as a measure of emotional arousal and autonomic activation. Psychophysiology, 45(4), 602-607. doi:10.1111/j.1469-8986.2008.00654.x

Chatterjee, A., \& Vartanian, O. (2014). Neuroaesthetics. Trends in Cognitive Sciences, 18(7), 370-375. doi:https://doi.org/10.1016/j.tics.2014.03.003

Cordaro, D. T., Sun, R., Keltner, D., Kamble, S., Huddar, N., \& McNeil, G. (2018). Universals and Cultural Variations in 22 Emotional Expressions Across Five Cultures. Emotion, 18(1), 75-93. doi:10.1037/emo0000302

Cowen, A. S., Fang, X., Sauter, D., \& Keltner, D. (2020). What music makes us feel: At least 13 dimensions organize subjective experiences associated with music across different cultures. Proceedings of the National Academy of Sciences, 117(4), 1924. doi:10.1073/pnas.1910704117

Cowen, A. S., \& Keltner, D. (2017). Self-report captures 27 distinct categories of emotion bridged by continuous gradients. Proceedings of the National Academy of Sciences. doi:10.1073/pnas.1702247114

Craig, A. D. (2002). How do you feel? Interoception: the sense of the physiological condition of the body. Nature Reviews Neuroscience, 3, 655. doi:10.1038/nrn894

Critchley, H. D., Wiens, S., Rotshtein, P., Ohman, A., \& Dolan, R. J. (2004). Neural systems supporting interoceptive awareness. Nat Neurosci, 7(2), 189-195. doi:10.1038/nn1176

Cupchik, G. C., Vartanian, O., Crawley, A., \& Mikulis, D. J. (2009). Viewing artworks: Contributions of cognitive control and perceptual facilitation to aesthetic experience. Brain and Cognition, 70(1), 84-91. doi:https://doi.org/10.1016/j.bandc.2009.01.003

Damasio, A., \& Carvalho, G. B. (2013). The nature of feelings: evolutionary and neurobiological origins. Nature Reviews Neuroscience, 14(2), 143-152. Retrieved from http://dx.doi.org/10.1038/nrn3403

Ekman, P. (1992). An argument for basic emotions. Cognition \& Emotion, 6(3-4), 169-200. doi:10.1080/02699939208411068

Hari, R., Henriksson, L., Malinen, S., \& Parkkonen, L. (2015). Centrality of Social Interaction in Human Brain Function. Neuron, 88(1), 181-193. doi:https://doi.org/10.1016/i.neuron.2015.09.022

Hari, R., \& Kujala, M. V. (2009). Brain Basis of Human Social Interaction: From Concepts to Brain Imaging. Physiological Reviews, 89(2), 453-479. doi:10.1152/physrev.00041.2007

Heikkilä, T. T., Laine, O., Savela, J., \& Nummenmaa, L., December 4). . (2020). Onni: An online experiment platform for research (Version 1.0): Zenodo.

Henderson, J. M. (2003). Human gaze control during real-world scene perception. Trends in Cognitive Sciences, 7(11), 498-504. doi:https://doi.org/10.1016/j.tics.2003.09.006

Hudson, M., Seppälä, K., Putkinen, V., Sun, L., Glerean, E., Karjalainen, T., . . Nummenmaa, L. (2020). Dissociable neural systems for unconditioned acute and sustained fear. Neuroimage, 216, 116522. doi:https://doi.org/10.1016/j.neuroimage.2020.116522 
Inzlicht, M., Shenhav, A., \& Olivola, C. Y. (2018). The Effort Paradox: Effort Is Both Costly and Valued. Trends in Cognitive Sciences, 22(4), 337-349.

doi:https://doi.org/10.1016/j.tics.2018.01.007

Johnston, V. S. (2006). Mate choice decisions: the role of facial beauty. Trends in Cognitive Sciences, 10(1), 9-13. doi:https://doi.org/10.1016/i.tics.2005.11.003

Joordens, J. C. A., d’Errico, F., Wesselingh, F. P., Munro, S., de Vos, J., Wallinga, J., . . . Roebroeks, W. (2014). Homo erectus at Trinil on Java used shells for tool production and engraving. Nature, 518, 228. doi:10.1038/nature13962

https://www.nature.com/articles/nature13962\#supplementary-information

Kallio-Tavin, M., Fast, H., Heimonen, K., Pusa, T., \& Hari, R. (in press). Touched and moved by art: Towards a transdisciplinary discourse on human experience. Research in Art and Education Journal.

Kaplan, S. (1987). Aesthetics, Affect, and Cognition: Environmental Preference from an Evolutionary Perspective. Environment and Behavior, 19(1), 3-32. doi:10.1177/0013916587191001

Kragel, P. A., Knodt, A. R., Hariri, A. R., \& LaBar, K. S. (2016). Decoding Spontaneous Emotional States in the Human Brain. PLOS Biology, 14(9), e2000106. doi:10.1371/journal.pbio.2000106

Leder, H., Belke, B., Oeberst, A., \& Augustin, D. (2004). A model of aesthetic appreciation and aesthetic judgments. British Journal of Psychology, 95(4), 489-508. doi:https://doi.org/10.1348/0007126042369811

Leder, H., Gerger, G., Dressler, S. G., \& Schabmann, A. (2012). How Art Is Appreciated. Psychology of Aesthetics Creativity and the Arts, 6(1), 2-10. doi:10.1037/a0026396

Menninghaus, W., Wagner, V., Hanich, J., Wassiliwizky, E., Jacobsen, T., \& Koelsch, S. (2017). The Distancing-Embracing model of the enjoyment of negative emotions in art reception. Behavioral and Brain Sciences, 40. doi:10.1017/S0140525X17000309

Menninghaus, W., Wagner, V., Wassiliwizky, E., Schindler, I., Hanich, J., Jacobsen, T., \& Koelsch, S. (2019). What Are Aesthetic Emotions? Psychological Review, 126(2), 171-195. doi: $10.1037 /$ rev0000135

Mohammad, S., \& Kiritchenko, S. (2018, may). WikiArt Emotions: An Annotated Dataset of Emotions Evoked by Art, Miyazaki, Japan.

Nummenmaa, L., Glerean, E., Hari, R., \& Hietanen, J. K. (2014). Bodily maps of emotions. Proceedings of the National Academy of Sciences of the United States of America, 111(2), 646-651. doi:10.1073/pnas.1321664111

Nummenmaa, L., Hari, R., Hietanen, J. K., \& Glerean, E. (2018). Maps of subjective feelings. Proceedings of the National Academy of Sciences of the United States of America, 115, 9198-9203.

Nummenmaa, L., Hietanen, J. K., Santtila, P., \& Hyona, J. (2012). Gender and Visibility of Sexual Cues Influence Eye Movements While Viewing Faces and Bodies. Archives of Sexual Behavior, 41(6), 1439-1451. doi:10.1007/s10508-012-9911-0

Nummenmaa, L., Hyona, J., \& Calvo, M. G. (2006). Eye movement assessment of selective attentional capture by emotional pictures. Emotion, 6(2), 257-268. doi:10.1037/15283542.6.2.257

Nummenmaa, L., \& Saarimäki, H. (2017). Emotions as discrete patterns of systemic activity. Neuroscience Letters. doi:https://doi.org/10.1016/j.neulet.2017.07.012

Nummenmaa, L., \& van Dillen, L. (2021). Carnal pleasures. Current Opinion in Behavioral Sciences, 39, 85-92. doi:https://doi.org/10.1016/j.cobeha.2021.02.026

Panksepp, J. (1982). Toward a general psychobiological theory of emotions. Behavioral and Brain Sciences, 5(03), 407-422. doi:doi:10.1017/S0140525X00012759

Pannasch, S., \& Velichkovsky, B. M. (2009). Distractor effect and saccade amplitudes: Further evidence on different modes of processing in free exploration of visual images. Visual Cognition, 17(6-7), 1109-1131. doi:10.1080/13506280902764422 
Pihko, E., Virtanen, A., Saarinen, V.-M., Pannasch, S., Hirvenkari, L., Tossavainen, T., . . Hari, R. (2011). Experiencing Art: The Influence of Expertise and Painting Abstraction Level. Frontiers in Human Neuroscience, 5, 94. doi:10.3389/fnhum.2011.00094

Putkinen, V., Nazari-Farsani, S., Seppälä, K., Karjalainen, T., Sun, L., Karlsson, H. K., . . . Nummenmaa, L. (2021). Decoding music-evoked emotions in the auditory and motor cortex. Cerebral Cortex, 2020.2005.2024.101667. doi:10.1101/2020.05.24.101667

Saarimäki, H., Ejtehadian, L. F., Glerean, E., Jaaskelainen, I. P., Vuilleumier, P., Sams, M., \& Nummenmaa, L. (2018). Distributed affective space represents multiple emotion categories across the human brain. Social Cognitive and Affective Neuroscience, 13, 471-482. doi:10.1093/scan/nsy018

Saarimäki, H., Gotsopoulos, A., Jääskeläinen, I. P., Lampinen, J., Vuilleumier, P., Hari, R., . . . Nummenmaa, L. (2016). Discrete Neural Signatures of Basic Emotions. Cerebral Cortex, 6, 2563-2573. doi:10.1093/cercor/bhv086

Skov, M., \& Nadal, M. (2020). There Are No Aesthetic Emotions: Comment on Menninghaus et al. (2019). Psychological Review, 127(4), 640-649. doi:10.1037/rev0000187

Spence, C., Okajima, K., Cheok, A. D., Petit, O., \& Michel, C. (2016). Eating with our eyes: From visual hunger to digital satiation. Brain and Cognition, 110, 53-63. doi:https://doi.org/10.1016/j.bandc.2015.08.006

Volynets, S., Glerean, E., Hietanen, J. K., Hari, R., \& Nummenmaa, L. (2019). Bodily maps of emotions are culturally universal. Emotion (W ashington, D.C.). doi:10.1037/emo0000624

Wager, T. D., Kang, J., Johnson, T. D., Nichols, T. E., Satpute, A. B., \& Barrett, L. F. (2015). A Bayesian Model of Category-Specific Emotional Brain Responses. PLOS Computational Biology, 11(4), e1004066. doi:10.1371/journal.pcbi.1004066

Zentner, M., Grandjean, D., \& Scherer, K. R. (2008). Emotions evoked by the sound of music: Characterization, classification, and measurement. Emotion, 8(4), 494-521.

doi:10.1037/1528-3542.8.4.494 
Table S-1 List of rating dimensions for the artworks and corresponding means and standard deviations

\begin{tabular}{lcc}
\hline Dimension & Mean & SD \\
\hline Sensory aesthetic evaluation & 54.47 & 11.23 \\
How much do you like this work? & 82.25 & 7.61 \\
Do you think this work is art? & 37.32 & 12.83 \\
How much do you have to struggle to understand this work? & 44.35 & 10.28 \\
How much do you empathize with this work? & 56.30 & 11.43 \\
How beautiful this work is? & 65.87 & 8.15 \\
How balanced this work is? & 54.91 & 11.14 \\
How elegant this work is? & & \\
& & \\
Emotional aesthetic evaluation & 46.66 & 13.23 \\
How touched do you feel while viewing the work? & 17.98 & 9.84 \\
How much sublimity do you feel while viewing the work? & 26.22 & 8.92 \\
How much amazement do you feel while viewing the work? & 41.40 & 9.57 \\
How much excitement do you feel while viewing the work? & 37.84 & 10.47 \\
How much moved do you feel while viewing the work? & & \\
\hline Basic emotional evaluation & 54.87 & 11.15 \\
How much joy do you feel while viewing the work? & 11.85 & 13.85 \\
How much sadness do you feel while viewing the work? & & 9.79 \\
How much surprise do you feel while viewing the work? & & 7.91 \\
How much fear do you feel while viewing the work? & & \\
How much anger do you feel while viewing the work? & & \\
How much disgust do you feel while viewing the work? & & \\
\hline & & \\
\hline
\end{tabular}


Table S-2 Correlations (Pearson) between mental and bodily experiences and aesthetic and affective dimensions of art. The data are sorted per correlation with bodily experience.

\begin{tabular}{lcc}
\hline Dimension & $\begin{array}{c}\text { Bodily } \\
\text { experience }\end{array}$ & $\begin{array}{c}\text { Mental } \\
\text { experience }\end{array}$ \\
\hline Touching & $\mathbf{0 . 2 6}$ & 0.04 \\
Empathizing & $\mathbf{0 . 2 2}$ & 0.07 \\
Moving & $\mathbf{0 . 2 0}$ & 0.05 \\
Sadness & $\mathbf{0 . 1 8}$ & 0.03 \\
Fear & $\mathbf{0 . 1 7}$ & 0.06 \\
Beauty & $\mathbf{0 . 1 5}$ & -0.03 \\
Art & $\mathbf{0 . 1 4}$ & 0.03 \\
Anger & $\mathbf{0 . 1 3}$ & -0.04 \\
Elegance & $\mathbf{0 . 1 2}$ & -0.01 \\
Liking & $\mathbf{0 . 1 1}$ & 0.06 \\
Sublimity & 0.08 & 0.02 \\
Disgust & 0.06 & 0.06 \\
Joy & 0.06 & 0.03 \\
Excitement & 0.05 & $\mathbf{0 . 1 6}$ \\
Balance & 0.02 & 0.05 \\
Surprise & -0.08 & $\mathbf{0 . 1 4}$ \\
Amazement & -0.12 & $\mathbf{0 . 1 8}$ \\
Effort & -0.22 & 0.08 \\
\hline
\end{tabular}



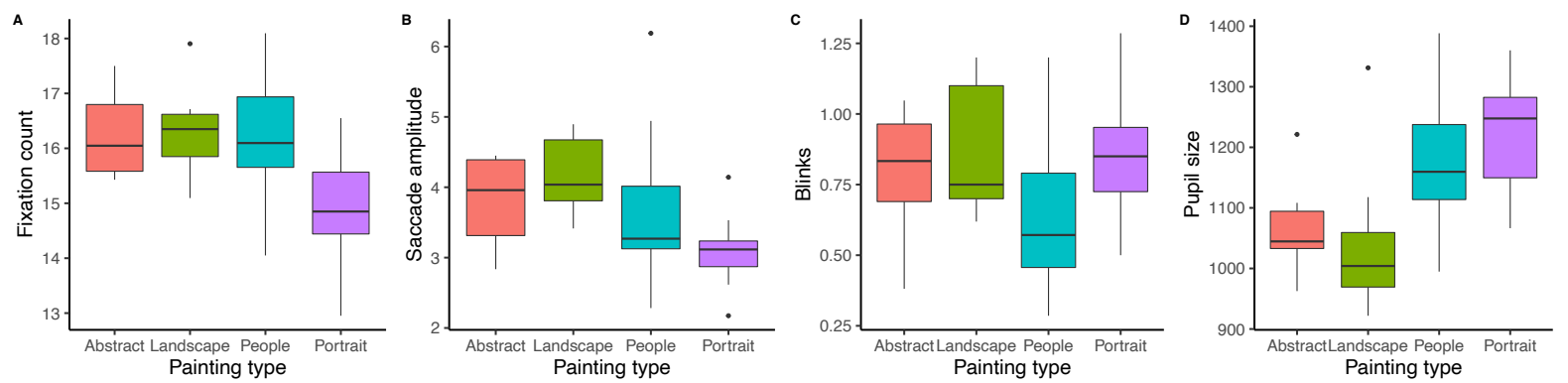

Figure S-1. Distributions of the eye movement parameters as a function of painting category. Horizontal line shows median, box interquartile range (IQR), and whiskers 1.5 * IQR. A) Number of fixations, B) Mean saccade amplitude (degrees), C) Number of blinks and D) Pupil size (arbitrary unit). 